\title{
Zur Feinstruktur von Ramie
}

\section{Doctoral Thesis}

\section{Author(s):}

Vogel, Alfred

Publication date:

1953

Permanent link:

https://doi.org/10.3929/ethz-a-000090038

Rights / license:

In Copyright - Non-Commercial Use Permitted 
Prom. Nr. 2274

\title{
Zur Feinstruktur von Ramie
}

\author{
von der \\ EIDGENÖSSISCHEN TECHNISCHEN \\ HOCHSCHULE IN ZURICH \\ zur Erlangung \\ der Würde eines Doktors der \\ Naturwissenschaften \\ genehmigte
}

PROMOTIONSARBEIT

vorgelegt von

\section{ALFRED VOGEL}

dipl. Naturwissenschafter von Kölliken (AG.)

Referent: Herr Professor Dr. A. Frey-Wyssling

Korreferent: Herr Professor Dr. H. Hopff

DR. ALFRED HÜTHIG VERLAG - HEIDELBERG 


\title{
Zur Feinstruktur von Ramie
}

\author{
von Alfred Vogel \\ Aus dem Institut für Allgemeine Botanik \\ der Eidgenössischen Technischen Hochschule Zürich
}

Vorsteher: Prof. Dr. A. Frey-Wyssling

\section{ZUSAMMENFA S UNG:}

Gereinigte Ramiefasern wurden auf verschiedene Weise mechanisch zertrümmert. In doppelt beschatteten elektronenmikroskopischen Präparaten wurden durchschnittliche Mikrofibrillenbreiten von 173-203 $\AA$ gemessen. Die Dicke der Mikrofibrillen, die mit ihrer Breitseite der Unterlage aufliegen, beträgt et wa 30 Å. Bei der Hydrolyse in $\mathrm{H}_{2} \mathrm{SO}_{4}$ werden die Fibrillen zu unregelmäßigen „Hydrolyseteilchen“ quergespalten. Diese sind aus kleineren Partikeln von $80-100 \AA$ Breite und $300-1000 \AA$ Å Länge zusammengesetzt, die wahrscheinlich den röntgenometrischen Micellen entsprechen.

\section{SUMMARY:}

Purified ramie-fibres were mechanically disintegrated. The double shadowed electron microscopic preparations revealed microfibrils with an average width of 173-203 $\AA$ and a thickness of about $30 \AA$. Their flattened side lays parallel to the supporting membrane. Hydrolysis in $\mathrm{H}_{2} \mathrm{SO}_{4}$ caused a breakage of the microfibrils into irregular "hydrolysis particles", which consist of smaller units with $80-100 \AA$ width and $300-1000 \AA$ length. These probably correspond to the micellae revealed by s-ray analysis.

\section{Einleitung}

Die Untersuchung der Feinstruktur von Zellulosefasern mit Hilfe der indirekten Methoden führte zu Fasermodellen (Frey-Wyssling 1936) ${ }^{5}$ ), die allen beobachteten Tatsachen gerecht wurden. Da sich mit der Einführung der Elektronenmikroskopie eine Möglichkeit ergab, die Fasermodelle zu verifizieren und zu ergänzen, war es naheliegend, daß sich die Forscher das neue Hilfsmittel rasch dienstbar machten und dazu zahlreiche Präparationsmethoden entwickelten.

E. Kuhn $(1941)^{21}$ ) quoll Schnitte von Zellulosefasern in Kupferoxydammoniak und stellte daraus durch Pressen Präparate her, die genügend dünn für die elektronenmikroskopischen Untersuchungen waren. Er beschrieb fibrilläre Strukturen, wobei er für die Dicke der Stränge 100 bis

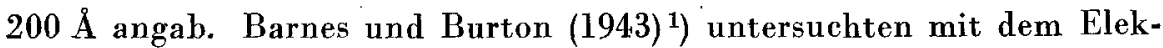
tronenmikroskop mechanisch zertrümmerte Baumwollfasern. 